\title{
Effect of time-odd mean fields on inertial parameters of the quadrupole collective Hamiltonian
}

\author{
N. Hinohara, ${ }^{1,2}$ Z. P. Li,${ }^{3,4}$ T. Nakatsukasa, ${ }^{2}$ T. Nikšić, ${ }^{4}$ and D. Vretenar ${ }^{4}$ \\ ${ }^{1}$ Department of Physics and Astronomy, University of North Carolina, Chapel Hill, North Carolina 27599-3255 \\ ${ }^{2}$ Theoretical Nuclear Physics Laboratory, RIKEN Nishina Center, RIKEN, Wako, Saitama 351-0198, Japan \\ ${ }^{3}$ School of Physical Science and Technology, Southwest University, Chongqing 400715, China \\ ${ }^{4}$ Physics Department, Faculty of Science, University of Zagreb, 10000 Zagreb, Croatia
}

(Received 13 December 2011; published 28 February 2012)

\begin{abstract}
Most current implementations of the quadrupole collective Hamiltonian model do not include the contributions of time-odd mean fields to the moments of inertia and mass parameters (Thouless-Valatin dynamical rearrangement contributions). A hybrid model is introduced that allows a quantitative estimate of these contributions to the inertial functions of a five-dimensional collective Hamiltonian based on microscopic energy density functionals. Fully self-consistent constrained relativistic Hartree-Bogoliubov calculations of triaxial energy surfaces in the $\beta-\gamma$ plane are used to determine the parameters of an equivalent pairing-plus-quadrupole $(P+Q)$ Hamiltonian. This Hamiltonian is employed in constrained Hartree-Fock-Bogoliubov (CHFB) plus local quasiparticle random-phase approximation (LQRPA) calculation of the deformation-dependent corrections to the Inglis-Belyaev moments of inertia and cranking mass parameters. This hybrid model is used to evaluate the influence of time-odd mean fields on vibrational and rotational collective masses and investigate their effect on the low-energy collective excitation spectra and transition rates of $\gamma$-soft Xe and Ba nuclei.
\end{abstract}

DOI: 10.1103/PhysRevC.85.024323

PACS number(s): 21.60.Jz, 21.60.Ev, 21.10.Re, 23.20.Lv

\section{INTRODUCTION}

The variation of nuclear ground-state shapes is governed by the modification of the shell structure of single-nucleon orbitals. Far from the $\beta$-stability line, in particular, the energy spacings between single-nucleon levels change considerably with the number of neutrons and/or protons. The reduction of spherical shell closure is often associated with the occurrence of deformed ground states and, in many cases, with the phenomenon of coexistence of different shapes in a single nucleus. A quantitative description of the evolution of nuclear shapes, including regions of short-lived exotic nuclei that are becoming accessible in experiments at radioactive-beam facilities, necessitate accurate modeling of the underlying microscopic nucleonic dynamics. Major advances in nuclear theory have recently been made in studies of complex shapes and the corresponding excitation spectra and electromagnetic decay patterns, especially in the framework of nuclear energy density functionals (EDFs) [1-4].

A microscopic, EDF-based description of complex collective excitation spectra usually starts from a constrained Hartree-Fock plus BCS (HFBCS) or Hartree-FockBogoliubov (HFB) calculation of the binding energy surface with the mass multipole moments as constrained quantities. The static nuclear mean field is characterized by symmetry breaking - translational, rotational, particle number. Even though symmetry breaking incorporates important static correlations (i.e., deformations and pairing), the static self-consistent solution can only provide an approximate description of bulk ground-state properties such as masses and radii. Modeling excitation spectra and transition rates in the EDF framework necessitates a systematic treatment of dynamical effects related to restoration of broken symmetries and fluctuations in collective coordinates.

In recent years several accurate and efficient models and algorithms, based on microscopic density functionals or effec- tive interactions, were developed that perform the restoration of symmetries broken by the static nuclear mean field and take into account quadrupole fluctuations. Many interesting phenomena related to shell evolution were investigated by employing the angular-momentum-projected generator coordinate method (GCM) with the axial quadrupole moment as the generating coordinate and with intrinsic configurations calculated in the HFB model with the finite-range Gogny interaction [5-9]. Recently this approach was extended to include full triaxial angular-momentum and particle-number projection [10]. Another sophisticated structure model that takes into account collective correlations is based on axially constrained HFBCS calculations with Skyrme effective interactions in the particle-hole channel and a density-dependent contact force in the pairing channel [11-15]. The latest extension of this model that incorporates triaxial angularmomentum projection was reported by the authors of Ref. [16]. The framework of relativistic EDFs has also been extended to include correlations related to the restoration of broken symmetries and to fluctuations of collective variables [17-20].

GCM configuration mixing of axially symmetric states is routinely used in nuclear structure studies, but the application of this method to triaxial shapes presents a much more involved and technically difficult problem. In addition, the use of general EDFs with an arbitrary dependence on nucleon densities in GCM-type calculations often leads to discontinuities or even divergences of the energy kernels as a function of deformation [21-23]. Only for a specific type of density dependence can a regularization method be implemented $[24,25]$ that corrects the energy kernels and removes the discontinuities and divergences.

In an alternative approach to five-dimensional quadrupole dynamics that restores rotational symmetry and allows for fluctuations around the triaxial mean-field minima, a collective Hamiltonian can be formulated, with deformation-dependent 
inertia parameters determined by microscopic self-consistent mean-field calculations. There are two principal approaches to derive the collective Hamiltonian starting from a microscopic framework based on an effective internucleon interaction or EDF. One is the GCM with the Gaussian overlap approximation (GOA) [26-28]. With the assumption that the GCM overlap kernels can be approximated by Gaussian functions [29], the local expansion of the kernels up to second order in the nonlocality transforms the GCM Hill-Wheeler equation into a second-order differential equation-the Schrödinger equation for the collective Hamiltonian. The kinetic part of this Hamiltonian contains an inertia tensor [30], and the potential energy is determined by the diagonal elements of the Hamiltonian kernel and also includes zero-point energy (ZPE) corrections [31].

Another microscopic derivation of the collective Hamiltonian is based on the adiabatic approximation to the timedependent HFB theory (ATDHFB) [32,33]. The adiabatic theories of the time-dependent mean field have been studied by many authors, resulting in different formulations. For instance, see Ref. [34] for historical and recent developments. To achieve a fully consistent local harmonic formulation it is necessary to take into account the curvature effect and lift the restriction to the point transformation for the canonical collective variables [35]. The gauge invariant formulation given by the authors of Ref. [36], based on the adiabatic self-consistent collective coordinate method [37], turns out to provide a consistent and practical method to simultaneously determine the optimal collective variables and the collective Hamiltonian. The collective potential and the mass parameters are determined by the constrained HFB equation and the local harmonic equations that are coupled with each other. The mass parameters are identical to the Thouless-Valatin masses [38] in the limit of vanishing curvatures that is valid at the local minima of the potential.

The dynamics of the collective Bohr Hamiltonian is governed by the vibrational inertial functions and the moments of inertia [39]. For these quantities either the GCM-GOA (Yoccoz masses [40]) or the ATDHFB expressions (Thouless-Valatin masses [38]) can be used. The Thouless-Valatin masses have the advantage that they also include the time-odd components of the mean-field potential and, in this sense, the full dynamics of a nuclear system. For instance, the Thouless-Valatin masses respect Galilean invariance and thus yield the exact translational mass. In contrast, generally neither the cranking nor the Yoccoz masses can reproduce the exact total mass. In the GCM approach these time-odd components can only be included properly if, in addition to the coordinates $q_{i}$, the corresponding canonically conjugate momenta $p_{i}$ are also taken into account, but this is obviously a very complicated task. In many applications a further simplification is introduced by adopting the cranking formulas $[31,41]$ that represent the unperturbative limit for the Thouless-Valatin masses and the corresponding expressions for ZPE corrections. This approximation was applied in recent studies using models based both on the Gogny interaction [42,43] and Skyrme EDFs [28,44]. The Thouless-Valatin corrections to the cranking mass formula are often treated by a simple phenomenological scaling with a factor of 1.2-1.4 [44]. In a recent systematic study [45] of low-energy nuclear structure at normal deformation, based on the HFB theory extended by the GCM and mapped onto a five-dimensional collective quadrupole Hamiltonian, the Thouless-Valatin moments of inertia were used, whereas the cranking approximation was still used for the quadrupole mass parameters. Using the Gogny $D 1 S$ interaction, even-even nuclei with proton numbers $Z=10$ to $Z=110$ and neutron numbers $N \leqslant 200$ were calculated.

In this work we consider a recent implementation for the solution of the eigenvalue problem of a five-dimensional collective Hamiltonian for quadrupole vibrational and rotational degrees of freedom, with parameters determined by constrained self-consistent relativistic Hartree-Bogoliubov calculations for triaxial shapes [46-51]. In the present implementation of the collective Hamiltonian model, however, the moments of inertia and mass parameters do not include the contributions of time-odd mean fields (the so-called Thouless-Valatin dynamical rearrangement contributions) and this breaks the self-consistency of the approach. Initial applications have shown that both the moments of inertia and mass parameters are systematically smaller than the empirical values and therefore they have to be renormalized to obtain a quantitative agreement with the data. A study in an exactly solvable model [52] has also shown the importance of including the time-odd mean fields in the description of collective dynamics.

Very recently an efficient microscopic method of deriving the five-dimensional quadrupole collective Hamiltonian was developed [53] based on the adiabatic self-consistent collective coordinate approach. In this method the vibrational and rotational collective masses (inertial functions) were determined by local normal modes built on constrained HFB states. Since the only input of the constrained HFB (CHFB) plus local quasiparticle random-phase approximation (LQRPA) method [53] was the microscopic Hamiltonian, this method may be used in conjunction with any effective interaction or energy density functional. So far only the pairing-plus-quadrupole $(P+Q)$ Hamiltonian has been used in the construction of the five-dimensional collective Hamiltonian using the CHFB + LQRPA method. However, since the parameters of the $P+Q$ Hamiltonian and quadrupole-pairing interaction are not universal this limits the application of the CHFB + LQRPA model.

Our aim is to construct a five-dimensional collective Hamiltonian based on the universal EDF framework, with the contribution of time-odd mean fields included in a selfconsistent way. As the first step, in this work we introduce a hybrid model that allows us to estimate the ThoulessValatin contributions to the moments of inertia and mass parameters, and analyze their effect on the collective excitation spectra of $\gamma$-soft $\mathrm{Xe}$ and $\mathrm{Ba}$ nuclei. The model utilizes the CHFB + LQRPA method, based on the $P+Q$ Hamiltonian, to calculate the deformation-dependent contributions of timeodd fields to the inertial functions. Section II includes a short review of the theoretical framework (the quadrupole collective Hamiltonian in five dimensions) and the CHFB + LQRPA method is outlined in Sec. III. The contribution of time-odd mean fields to the inertial functions and their effect on lowenergy collective spectra is analyzed in Sec. IV. Finally Sec. V 
summarizes the results and presents an outlook for future studies.

\section{QUADRUPOLE COLLECTIVE HAMILTONIAN IN FIVE DIMENSIONS}

\section{A. Collective Hamiltonian}

The general Bohr collective model for the description of quadrupole collective states, including a detailed discussion of the model's kinematics, was recently reviewed by the authors of Ref. [28]. Nuclear excitations determined by quadrupole vibrational and rotational degrees of freedom can be treated simultaneously by considering five quadrupole collective coordinates $\alpha_{\mu}, \mu=-2,-1, \ldots, 2$ that describe the surface of a deformed nucleus: $R=R_{0}\left[1+\sum_{\mu} \alpha_{\mu} Y_{2 \mu}^{*}\right]$. To separate rotational and vibrational motion these coordinates are usually parameterized in terms of two deformation parameters $\beta$ and $\gamma$ and three Euler angles $(\phi, \theta, \psi) \equiv \Omega$ that define the orientation of the intrinsic principal axes in the laboratory frame

$$
\alpha_{\mu}=D_{\mu 0}^{2}(\Omega) \beta \cos \gamma+\frac{1}{\sqrt{2}}\left[D_{\mu 2}^{2}(\Omega)+D_{\mu-2}^{2}(\Omega)\right] \beta \sin \gamma,
$$

where $D_{\mu \nu}^{\lambda}$ is the Wigner function. The three terms of the classical collective Hamiltonian, expressed in terms of the intrinsic variables $\beta, \gamma$, and Euler angles

$$
H_{\text {coll }}=\mathcal{T}_{\text {vib }}(\beta, \gamma)+\mathcal{T}_{\text {rot }}(\beta, \gamma, \Omega)+\mathcal{V}_{\text {coll }}(\beta, \gamma),
$$

denote the contributions from the vibrational kinetic energy

$$
\mathcal{T}_{\text {vib }}=\frac{1}{2} B_{\beta \beta} \dot{\beta}^{2}+\beta B_{\beta \gamma} \dot{\beta} \dot{\gamma}+\frac{1}{2} \beta^{2} B_{\gamma \gamma} \dot{\gamma}^{2},
$$

the rotational kinetic energy

$$
\mathcal{T}_{\text {rot }}=\frac{1}{2} \sum_{k=1}^{3} \mathcal{I}_{k} \omega_{k}^{2},
$$

and the collective potential energy $\mathcal{V}_{\text {coll }}(\beta, \gamma)$. The mass parameters $B_{\beta \beta}, B_{\beta \gamma}, B_{\gamma \gamma}$, and the moments of inertia $\mathcal{I}_{k}$ depend on the quadrupole deformation variables $\beta$ and $\gamma$.

In general, the classical kinetic energy for the coordinates $q_{i}$ with a coordinate-dependent mass tensor $B_{i j}(q)$

$$
\mathcal{T}_{\text {vib }}+\mathcal{T}_{\text {rot }}=\frac{1}{2} \sum_{i j} B_{i j}(q) \dot{q}_{i} \dot{q}_{j},
$$

can be quantized, following Pauli's prescription [54], as

$$
\hat{T}=-\frac{\hbar^{2}}{2} \frac{1}{\sqrt{\operatorname{det} B}} \sum_{i j} \frac{\partial}{\partial q_{i}} \sqrt{\operatorname{det} B}\left(B^{-1}\right)_{i j} \frac{\partial}{\partial q_{j}} .
$$

In the present model of Eq. (2) the kinetic energy tensor takes the block diagonal form

$$
B=\left(\begin{array}{cc}
B_{\mathrm{vib}} & 0 \\
0 & B_{\mathrm{rot}}
\end{array}\right),
$$

with the vibrational part of the tensor

$$
B_{\text {vib }}=\left(\begin{array}{cc}
B_{\beta \beta} & \beta B_{\beta \gamma} \\
\beta B_{\beta \gamma} & \beta^{2} B_{\gamma \gamma}
\end{array}\right) .
$$

In general, the rotational part is a complicated function of the Euler angles, but using the quasicoordinates related to the components of the angular momentum in the body-fixed frame, it takes a simple diagonal form

$$
\left(B_{\mathrm{rot}}\right)_{i k}=\delta_{i k} \mathcal{I}_{k}, \quad k=1,2,3,
$$

with the moments of inertia expressed as

$$
\mathcal{I}_{k}=4 B_{k} \beta^{2} \sin ^{2}(\gamma-2 k \pi / 3) .
$$

This particular functional form implies that all three moments of inertia vanish for the spherical configuration $(\beta=0)$ and, additionally, $\mathcal{I}_{z}$ and $\mathcal{I}_{y}$ vanish for axially symmetric prolate $\left(\gamma=0^{\circ}\right)$ and oblate $\left(\gamma=60^{\circ}\right)$ configurations, respectively. Note that hereafter we use the notation $\left(\mathcal{I}_{x}, \mathcal{I}_{y}, \mathcal{I}_{z}\right)$ and $\left(B_{x}, B_{y}, B_{z}\right)$. The resulting determinant reads

$$
\operatorname{det} B=\operatorname{det} B_{\mathrm{vib}} \cdot \operatorname{det} B_{\mathrm{rot}}=4 w r \beta^{8} \sin ^{2} 3 \gamma,
$$

where $w=B_{\beta \beta} B_{\gamma \gamma}-B_{\beta \gamma}^{2}$ and $r=B_{x} B_{y} B_{z}$. The quantized collective Hamiltonian can hence be written in the form

$$
\hat{H}=\hat{T}_{\text {vib }}+\hat{T}_{\text {rot }}+\hat{V}_{\text {coll }},
$$

with

$$
\begin{aligned}
\hat{T}_{\text {vib }}= & -\frac{\hbar^{2}}{2 \sqrt{w r}}\left\{\frac { 1 } { \beta ^ { 4 } } \left[\frac{\partial}{\partial \beta} \sqrt{\frac{r}{w}} \beta^{4} B_{\gamma \gamma} \frac{\partial}{\partial \beta}\right.\right. \\
& \left.-\frac{\partial}{\partial \beta} \sqrt{\frac{r}{w}} \beta^{3} B_{\beta \gamma} \frac{\partial}{\partial \gamma}\right] \\
& +\frac{1}{\beta \sin 3 \gamma}\left[-\frac{\partial}{\partial \gamma} \sqrt{\frac{r}{w}} \sin 3 \gamma B_{\beta \gamma} \frac{\partial}{\partial \beta}\right. \\
& \left.\left.+\frac{1}{\beta} \frac{\partial}{\partial \gamma} \sqrt{\frac{r}{w}} \sin 3 \gamma B_{\beta \beta} \frac{\partial}{\partial \gamma}\right]\right\},
\end{aligned}
$$

and

$$
\hat{T}_{\text {rot }}=\frac{1}{2} \sum_{k=1}^{3} \frac{\hat{J}_{k}^{2}}{\mathcal{I}_{k}},
$$

where $\hat{J}_{k}$ denotes the components of the angular momentum in the body-fixed frame of a nucleus. $\hat{V}_{\text {coll }}$ is the collective potential. The Hamiltonian describes quadrupole vibrations, rotations, and the coupling of these collective modes. The determinant Eq. (11) determines the volume element in the collective space

$$
\int d \tau_{\text {coll }}=\int_{0}^{\infty} d \beta \beta^{4} \int_{0}^{\pi / 3} d \gamma|\sin 3 \gamma| \int d \Omega \sqrt{w r},
$$

and the quantized Hamiltonian Eq. (12) is Hermitian with respect to the collective measure Eq. (15).

The diagonalization of the Hamiltonian yields the excitation energies and collective wave functions

$$
\Psi_{\alpha}^{J M}(\beta, \gamma, \Omega)=\sum_{K \in \Delta J} \psi_{\alpha K}^{J}(\beta, \gamma) \Phi_{M K}^{J}(\Omega) .
$$



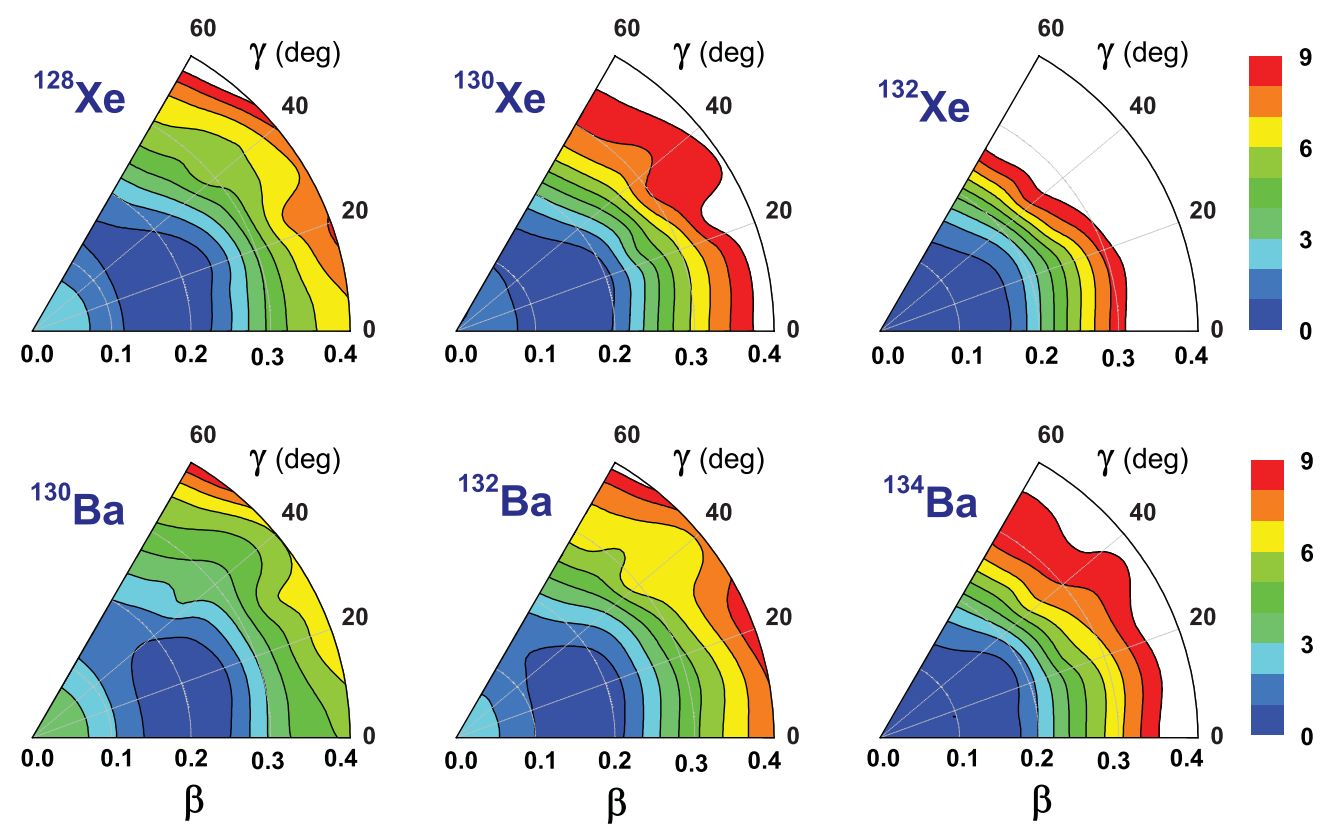

FIG. 1. (Color online) Self-consistent RHB triaxial quadrupole binding energy maps of even-even ${ }^{128-132}$ Xe and ${ }^{130-134} \mathrm{Ba}$ in the $\beta-\gamma$ plane $\left(0^{\circ} \leqslant \gamma \leqslant 60^{\circ}\right)$. All energies are normalized with respect to the corresponding minima. The energy gap between two neighboring contours is $1 \mathrm{MeV}$.

The angular part corresponds to a linear combination of Wigner functions

$$
\Phi_{M K}^{J}(\Omega)=\sqrt{\frac{2 J+1}{16 \pi^{2}\left(1+\delta_{K 0}\right)}}\left[D_{M K}^{J *}(\Omega)+(-1)^{J} D_{M-K}^{J *}(\Omega)\right],
$$

and the summation in Eq. (16) is over the allowed set of the $K$ values

$$
\Delta J= \begin{cases}0,2, \ldots, J & \text { for } \quad J \bmod 2=0, \\ 2,4, \ldots, J-1 & \text { for } \quad J \bmod 2=1 .\end{cases}
$$

Using the collective wave functions Eq. (16), various observables can be calculated and compared with experimental results. For instance, the quadrupole $E 2$ reduced transition probability

$$
B\left(E 2 ; \alpha J \rightarrow \alpha^{\prime} J^{\prime}\right)=\frac{1}{2 J+1}\left|\left\langle\alpha^{\prime} J^{\prime}|| \hat{\mathcal{M}}(E 2) \| \alpha J\right\rangle\right|^{2},
$$

where $\hat{\mathcal{M}}(E 2)$ is the electric quadrupole operator.

\section{B. Microscopic calculation: Potential and inertial functions}

The entire dynamics of the collective Hamiltonian is governed by the seven functions of the intrinsic deformations $\beta$ and $\gamma$ : the collective potential; the three mass parameters $B_{\beta \beta}, B_{\beta \gamma}$, $B_{\gamma \gamma}$; and the three moments of inertia $\mathcal{I}_{k}$. These functions are determined by the choice of a particular microscopic nuclear EDF or effective interaction. The map of the energy surface as function of the quadrupole deformation is obtained by imposing constraints on the axial and triaxial mass quadrupole moments. The quasiparticle wave functions and energies, generated from constrained self-consistent solutions, provide the microscopic input for the parameters of the collective Hamiltonian.

As an illustrative example, in Fig. 1 we display the quadrupole constrained self-consistent energy surfaces of the even-even nuclei ${ }^{128-132} \mathrm{Xe}$ and ${ }^{130-134} \mathrm{Ba}$ in the $\beta-\gamma$ plane $\left(0^{\circ} \leqslant \gamma \leqslant 60^{\circ}\right)$, calculated in the framework of the relativistic Hartree-Bogoliubov (RHB) model [3]. The calculation was performed using the relativistic functional $D D-P C 1$ [55] in the particle-hole channel. Pairing correlations are taken into account by an interaction separable in momentum space that was adjusted to reproduce the density dependence of the gap at the Fermi surface in nuclear matter, as calculated with a Gogny force [56,57]. All energies are normalized with respect to the binding energy of the absolute minimum and the contours join points on the surface with the same energy (in $\mathrm{MeV}$ ). Both for $\mathrm{Xe}$ and $\mathrm{Ba}$ these plots illustrate the rapid transition from $\gamma$-soft energy surfaces for lighter isotopes to spherical shapes near the $N=82$ closed shell.

The collective energy surface includes the energy of the zero-point motion, and this quantity has to be subtracted. The collective ZPE corresponds to a superposition of zero-point motion of individual nucleons in the single-nucleon potential. In the general case, the ZPE corrections on the potential energy surfaces depend on the deformation. The ZPE includes terms originating from the vibrational and rotational kinetic energy and a contribution of potential energy

$$
\Delta V\left(q_{0}, q_{2}\right)=\Delta V_{\mathrm{vib}}\left(q_{0}, q_{2}\right)+\Delta V_{\mathrm{rot}}\left(q_{0}, q_{2}\right)+\Delta V_{\mathrm{pot}}\left(q_{0}, q_{2}\right),
$$

where $q_{0}=\left\langle\hat{Q}_{20}\right\rangle$ and $q_{2}=\left\langle\hat{Q}_{22}\right\rangle$. These moments are related to the Hill-Wheeler coordinates $\beta(\beta>0)$ and $\gamma$ by the 
following relations:

$$
\begin{aligned}
& q_{20}=\sqrt{\frac{5}{16 \pi}}\left\langle 2 z^{2}-x^{2}-y^{2}\right\rangle=\frac{3}{4 \pi} A R_{0}^{2} \beta \cos \gamma, \\
& q_{22}=\sqrt{\frac{15}{32 \pi}}\left\langle x^{2}-y^{2}\right\rangle=\frac{3}{4 \pi} A R_{0}^{2} \frac{1}{\sqrt{2}} \beta \sin \gamma,
\end{aligned}
$$

where $R_{0}=1.2 A^{1 / 3} \mathrm{fm}$.

$\Delta V_{\text {pot }}\left(q_{0}, q_{2}\right)$ is much smaller than the contribution of the kinetic energy and is usually neglected [42]. Simple prescriptions for the calculation of vibrational and rotational ZPE were derived by the authors of Ref. [31]. Both corrections are calculated in the cranking approximation. The vibrational $\mathrm{ZPE}$ is given by the expression

$$
\Delta V_{\mathrm{vib}}\left(q_{0}, q_{2}\right)=\frac{1}{4} \operatorname{Tr}\left[\mathcal{M}_{(3)}^{-1} \mathcal{M}_{(2)}\right]
$$

The rotational ZPE is a sum of three terms:

$$
\begin{aligned}
& \Delta V_{\text {rot }}\left(q_{0}, q_{2}\right) \\
& \quad=\Delta V_{-2-2}\left(q_{0}, q_{2}\right)+\Delta V_{-1-1}\left(q_{0}, q_{2}\right)+\Delta V_{11}\left(q_{0}, q_{2}\right),
\end{aligned}
$$

with

$$
\Delta V_{\mu \nu}\left(q_{0}, q_{2}\right)=\frac{1}{4} \frac{\mathcal{M}_{(2), \mu \nu}\left(q_{0}, q_{2}\right)}{\mathcal{M}_{(3), \mu \nu}\left(q_{0}, q_{2}\right)} \quad \mu, v=0,2
$$

and

$$
\mathcal{M}_{(n), \mu \nu}\left(q_{0}, q_{2}\right)=\sum_{i, j} \frac{\left|\left\langle\Phi\left|\hat{Q}_{2 \mu}\right| i j\right\rangle\left\langle i j\left|\hat{Q}_{2 v}\right| \Phi\right\rangle\right|}{\left(E_{i}+E_{j}\right)^{n}} \quad \mu, v=0,2 .
$$

The individual terms are calculated from Eqs. (25) and (26), with the intrinsic components of the quadrupole operator defined by

$$
\begin{aligned}
& \hat{Q}_{20}=2 z^{2}-x^{2}-y^{2}, \quad \hat{Q}_{21}=-2 i y z, \quad \hat{Q}_{2-1}=-2 x z, \\
& \hat{Q}_{22}=x^{2}-y^{2}, \quad \hat{Q}_{2-2}=2 i x y .
\end{aligned}
$$

The potential $\hat{V}_{\text {coll }}$ in the collective Hamiltonian Eq. (12) is obtained by subtracting the ZPE corrections from the total mean-field energy

$$
\begin{aligned}
& V_{\text {coll }}\left(q_{0}, q_{2}\right) \\
& \quad=E_{\text {tot }}\left(q_{0}, q_{2}\right)-\Delta V_{\text {vib }}\left(q_{0}, q_{2}\right)-\Delta V_{\text {rot }}\left(q_{0}, q_{2}\right) .
\end{aligned}
$$

In the simplest approximation that does not break timereversal symmetry, the moments of inertia are calculated from the Inglis-Belyaev formula

$$
\mathcal{I}_{k}=\sum_{i, j} \frac{\left|\left\langle i j\left|\hat{J}_{k}\right| \Phi\right\rangle\right|^{2}}{E_{i}+E_{j}} \quad k=1,2,3,
$$

where $k$ denotes the axis of rotation, the summation runs over proton and neutron quasiparticle states $|i j\rangle=\beta_{i}^{\dagger} \beta_{j}^{\dagger}|\Phi\rangle$, and $|\Phi\rangle$ represents the quasiparticle vacuum. The mass parameters associated with the two quadrupole collective coordinates $q_{0}$ and $q_{2}$ are calculated in the cranking approximation

$$
B_{\mu \nu}\left(q_{0}, q_{2}\right)=\frac{\hbar^{2}}{2}\left[\mathcal{M}_{(1)}^{-1} \mathcal{M}_{(3)} \mathcal{M}_{(1)}^{-1}\right]_{\mu \nu} \quad \mu, v=0,2 .
$$

With the moments of inertia and mass parameters determined as described above, the diagonalization of the corresponding Hamiltonian yields collective excitation spectra and electromagnetic transition rates that qualitatively reproduce the data (cf. Sec. IV). A more detailed comparison, however, shows that the calculated excitation energies are, in general,
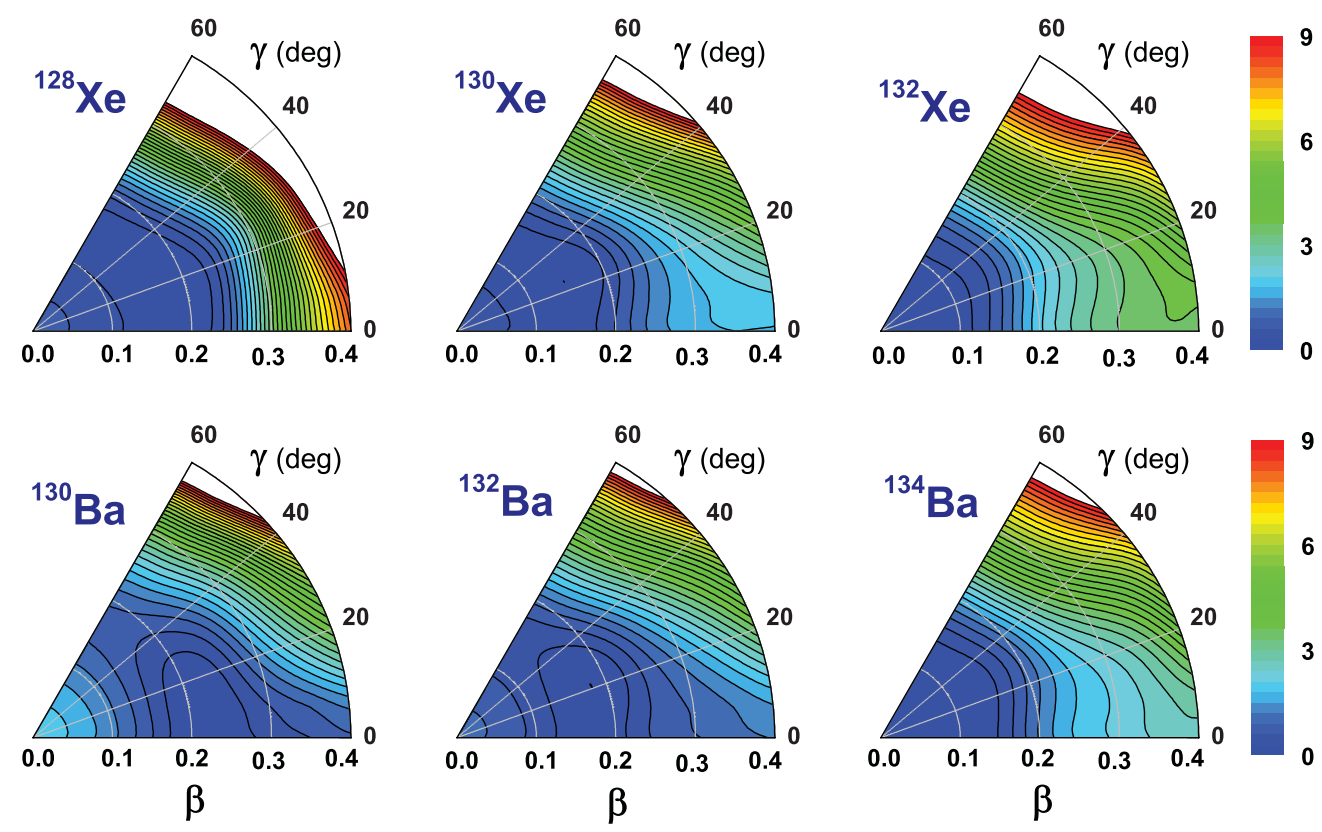

FIG. 2. (Color online) Quadrupole energy surfaces in the $\beta-\gamma$ plane $\left(0^{\circ} \leqslant \gamma \leqslant 60^{\circ}\right)$ of the even-even ${ }^{128-132} \mathrm{Xe}$ and ${ }^{130-134} \mathrm{Ba}$ isotopes, calculated with the pairing + quadrupole Hamiltonian (see text for description). All energies are normalized with respect to the corresponding absolute minimum. The energy gap between two neighboring contours is $0.3 \mathrm{MeV}$. 
considerably above their experimental counterparts, that is, the theoretical excitation spectrum is stretched compared with the experiment [50]. The reason is that the moments of inertia and mass parameters do not include the contributions of time-odd fields (the so-called Thouless-Valatin dynamical rearrangement contributions) and therefore are systematically smaller than the empirical values. In the case of well-deformed axially symmetric nuclei, several studies [42] showed that the Thouless-Valatin corrections are almost independent of deformation and the effective moments of inertia to be used in the collective Hamiltonian can simply be obtained by renormalizing the Inglis-Belyaev values by a constant factor, characteristic for a given nucleus. The situation is considerably more complicated in the case of mass parameters $[28,44]$ for which there are no simple estimates of the Thouless-Valatin correction, especially for nuclei with $\gamma$-soft potential energy surfaces. Some authors [28] argued that, to approximately take into account the Thouless-Valatin correction, all inertial functions, not only the moments of inertia, should be rescaled by a constant multiplicative factor.

In the following sections we introduce a hybrid model that allows us to estimate the Thouless-Valatin contributions to the moments of inertia and mass parameters and analyze their effect on the collective excitation spectra of $\gamma$-soft Xe and Ba nuclei.

\section{CONSTRAINED HARTREE-FOCK-BOGOLIUBOV PLUS LOCAL QUASIPARTICLE RANDOM-PHASE APPROXIMATION}

The Inglis-Belyaev (IB) formula Eq. (29) for the moments of inertia, and the cranking approximation Eq. (30) for the mass parameters of the collective Hamiltonian neglect the contribution of time-odd components of the moving mean field and therefore break the self-consistency of the theoretical framework [32,58]. It will be shown that the inclusion of these contributions leads to a significant increase of the vibrational and rotational collective masses. In the present study we employ the recently developed CHFB plus LQRPA method [53], based on the adiabatic self-consistent collective coordinate model [37].

In the CHFB + LQRPA approach the mass parameters and moments of inertia are evaluated using the local normal modes built on constrained mean-field states in the $(\beta, \gamma)$ deformation space. The CHFB equation is written in a variational form as

$$
\delta\left\langle\phi(\beta, \gamma)\left|\hat{H}_{\mathrm{CHFB}}(\beta, \gamma)\right| \phi(\beta, \gamma)\right\rangle=0,
$$
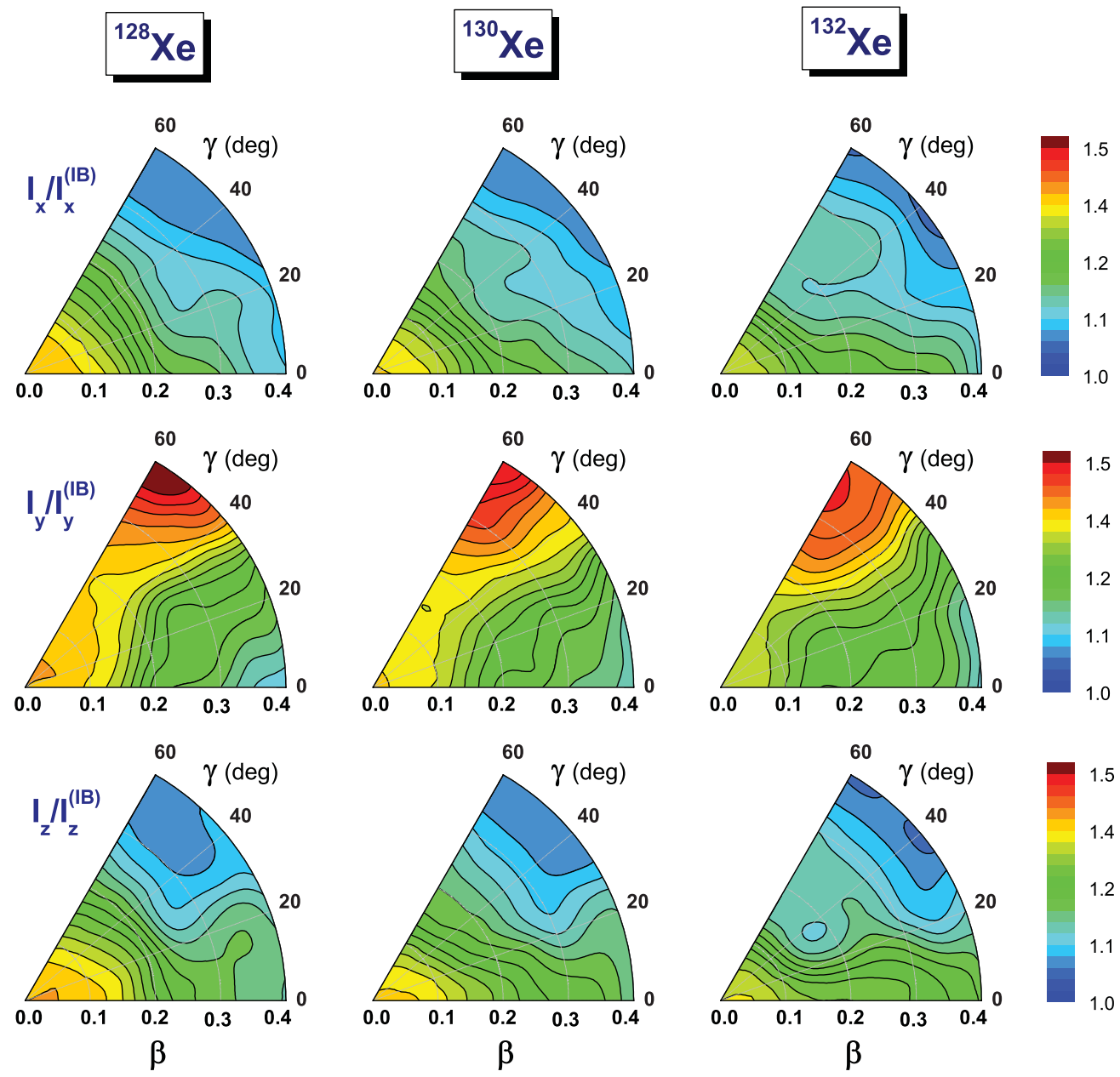

FIG. 3. (Color online) Ratios of the moments of inertia $B_{x}, B_{y}$, and $B_{z}$, calculated with the LQRPA and the IB formula, as functions of the deformations $\beta$ and $\gamma$, for the nuclei ${ }^{128-132} \mathrm{Xe}$. 
where the Hamiltonian $\hat{H}_{\mathrm{CHFB}}(\beta, \gamma)$ is defined as

$$
\begin{aligned}
& \hat{H}_{\mathrm{CHFB}}(\beta, \gamma) \\
& \quad=\hat{H}-\sum_{\tau=n, p} \lambda^{(\tau)}(\beta, \gamma) \widetilde{N}^{(\tau)}-\sum_{\mu=0,2} \kappa_{\mu}(\beta, \gamma) \hat{Q}_{2 \mu} .
\end{aligned}
$$

The constrained quantities are the numbers of neutrons and protons and the two mass quadrupole moments. $\hat{H}$ denotes a given microscopic Hamiltonian.

For each CHFB state in the $\beta-\gamma$ plane, the local normal modes are evaluated by solving the LQRPA equations

$$
\begin{gathered}
\delta\langle\phi(\beta, \gamma)|\left[\hat{H}_{\mathrm{CHFB}}(\beta, \gamma), \hat{Q}^{i}(\beta, \gamma)\right] \\
\quad-\frac{1}{i} \hat{P}_{i}(\beta, \gamma)|\phi(\beta, \gamma)\rangle=0, \\
\delta\langle\phi(\beta, \gamma)|\left[\hat{H}_{\mathrm{CHFB}}(\beta, \gamma), \frac{1}{i} \hat{P}_{i}(\beta, \gamma)\right] \\
\quad-C_{i}(\beta, \gamma) \hat{Q}^{i}(\beta, \gamma)|\phi(\beta, \gamma)\rangle=0 .
\end{gathered}
$$

Here $\hat{Q}^{i}(\beta, \gamma)$ and $\hat{P}_{i}(\beta, \gamma)$ are the QRPA infinitesimal generators and $C_{i}(\beta, \gamma)=\omega_{i}^{2}(\beta, \gamma)$ denotes the squared eigenfrequency of the normal mode. Among the LQRPA solutions the two collective are selected following the minimal metric criterion of the authors of Ref. [53]. The vibrational mass parameters $B_{\beta \beta}, B_{\gamma \gamma}$, and $B_{\beta \gamma}$ are then determined by the local transformation of the two collective coordinates $\left(q^{1}, q^{2}\right)$, spanned by the two collective LQRPA modes, into the quadrupole deformation space $(\beta, \gamma)$. The mass parameters associated with $q_{0}$ and $q_{2}$ are given by

$$
B_{\mu \nu}(\beta, \gamma)=\sum_{i=1,2} \frac{\partial q^{i}}{\partial q_{\mu}} \frac{\partial q^{i}}{\partial q_{v}} \quad \mu, v=0,2 .
$$

The partial derivatives are evaluated using the LQRPA infinitesimal generators

$$
\frac{\partial q_{\mu}}{\partial q^{i}}=\left\langle\phi(\beta, \gamma)\left|\left[\hat{Q}_{2 \mu}, \frac{1}{i} \hat{P}_{i}(\beta, \gamma)\right]\right| \phi(\beta, \gamma)\right\rangle .
$$

The rotational moments of inertia are calculated by solving the LQRPA equations for collective rotation at each of the CHFB states

$$
\begin{gathered}
\delta\langle\phi(\beta, \gamma)|\left[\hat{H}_{\mathrm{CHFB}}(\beta, \gamma), \hat{\Psi}_{k}(\beta, \gamma)\right] \\
-\frac{1}{i} \mathcal{I}_{k}^{-1}(\beta, \gamma) \hat{J}_{k}|\phi(\beta, \gamma)\rangle=0,
\end{gathered}
$$

together with the orthonormalization condition

$$
\left\langle\phi(\beta, \gamma)\left|\left[\hat{\Psi}_{k}(\beta, \gamma), \hat{J}_{k^{\prime}}\right]\right| \phi(\beta, \gamma)\right\rangle=i \delta_{k k^{\prime}},
$$

where $\hat{\Psi}_{k}(\beta, \gamma)$ and $\hat{J}_{k}$ denote the rotational angles and the angular momentum operators, respectively, corresponding to the three principal axes associated with the CHFB state $|\phi(\beta, \gamma)\rangle$. The resulting rotational moments of inertia present an extension of the Thouless-Valatin moments of inertia [38] for the equilibrium HFB state to nonequilibrium CHFB states.

The only input for a CHFB + LQRPA calculation is the microscopic Hamiltonian and therefore this method may be used in conjunction with any effective interaction of energy
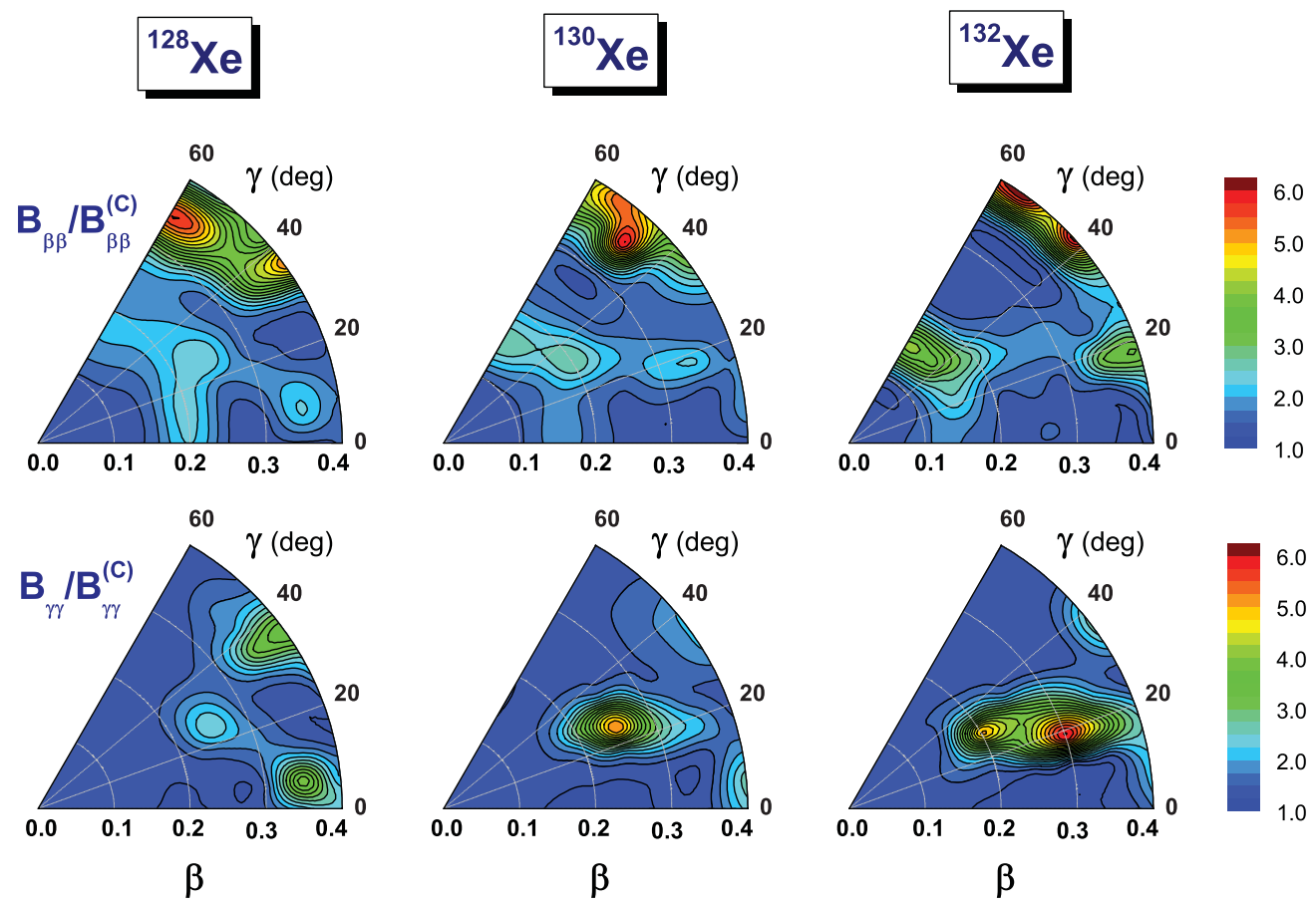

FIG. 4. (Color online) Ratios of the vibrational mass parameters $B_{\beta \beta}$ and $B_{\gamma \gamma}$ calculated with the LQRPA and in the cranking approximation, as functions of the deformations $\beta$ and $\gamma$, for the nuclei ${ }^{128-132} \mathrm{Xe}$. 
density functional. In applications so far the pairing-plusquadrupole $(P+Q)$ Hamiltonian, including the quadrupolepairing interaction, has been employed to construct the five-dimensional collective Hamiltonian with the use of the CHFB + LQRPA method $[53,59,60]$. This method was also applied to determine the collective Hamiltonian in the axially symmetric case, starting from the Skyrme energy density functional [61].

\section{EFFECT OF TIME-ODD MEAN FIELDS ON QUADRUPOLE COLLECTIVE DYNAMICS}

\section{A. Moments of inertia and vibrational masses}

The moments of inertia and vibrational mass parameters of the collective Hamiltonian introduced in Sec. II, based on a microscopic EDF, do not contain the ThoulessValatin contributions of the time-odd components of the self-consistent mean-field potential. To investigate their effect on the corresponding low-energy excitation spectra, here we employ a hybrid model that utilizes the CHFB + LQRPA method, based on the $P+Q$ Hamiltonian, to calculate the deformation-dependent corrections to the IB moments of inertia and cranking mass parameters.
The $P+Q$ Hamiltonian [62-67] used in CHFB + LQRPA model reads

$$
\begin{aligned}
\hat{H}= & \sum_{k \in \tau, \tau=(n, p)} \varepsilon_{k}^{(\tau)} \hat{c}_{k}^{(\tau) \dagger} \hat{c}_{k}^{(\tau)} \\
& -\sum_{\tau=(n, p)} \frac{G_{0}^{(\tau)}}{2}\left(\hat{A}^{(\tau) \dagger} \hat{A}^{(\tau)}+\hat{A}^{(\tau)} \hat{A}^{(\tau) \dagger}\right) \\
& -\sum_{\tau=(n, p)} \frac{G_{2}^{(\tau)}}{2} \sum_{K=-2}^{2}\left(\hat{B}_{2 K}^{(\tau) \dagger} \hat{B}_{2 K}^{(\tau)}+\hat{B}_{2 K}^{(\tau)} \hat{B}_{2 K}^{(\tau) \dagger}\right) \\
& -\frac{\chi}{2} \sum_{K=-2}^{2} \hat{D}_{2 K}^{\dagger} \hat{D}_{2 K} .
\end{aligned}
$$

It consists of a single-particle term, the monopole and quadrupole pairing interaction terms, and the quadrupolequadrupole interaction. $\left(\hat{A}^{(\tau)}, \hat{B}_{2 K}^{(\tau)}\right.$, and $\hat{D}_{2 K}$ are the monopole pairing, quadrupole pairing, and quadrupole particle-hole operators, respectively.) The model includes the following parameters: spherical single-particle energies $\varepsilon_{k}^{(\tau)}$, monopole and quadrupole pairing strengths $G_{J}^{(\tau)}$, and the strength parameter of the quadrupole-quadrupole interaction $\chi$. The inclusion of the quadrupole pairing is essential because
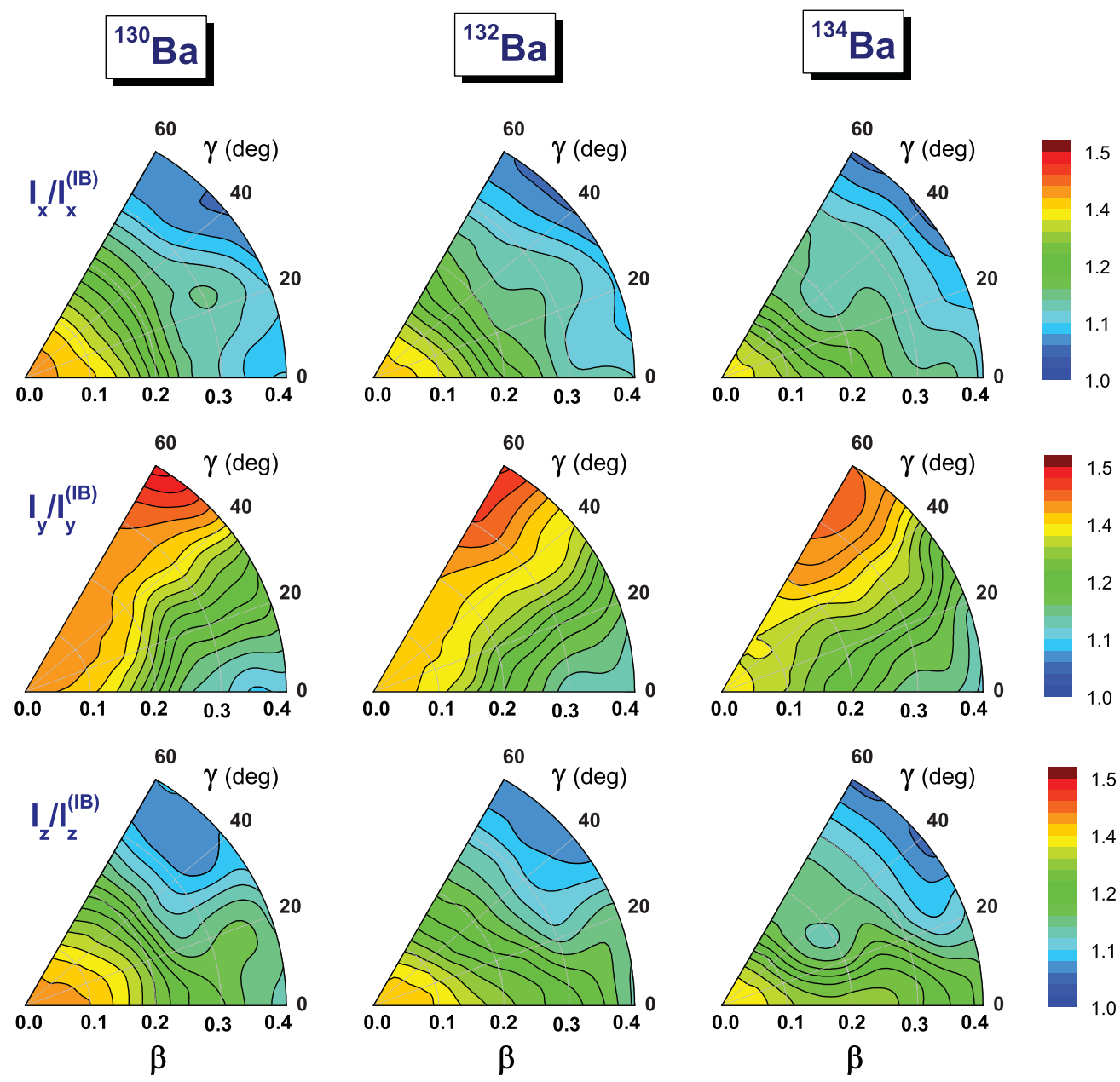

FIG. 5. (Color online) Same as described in the caption to Fig. 3 but for ${ }^{130-134} \mathrm{Ba}$. 

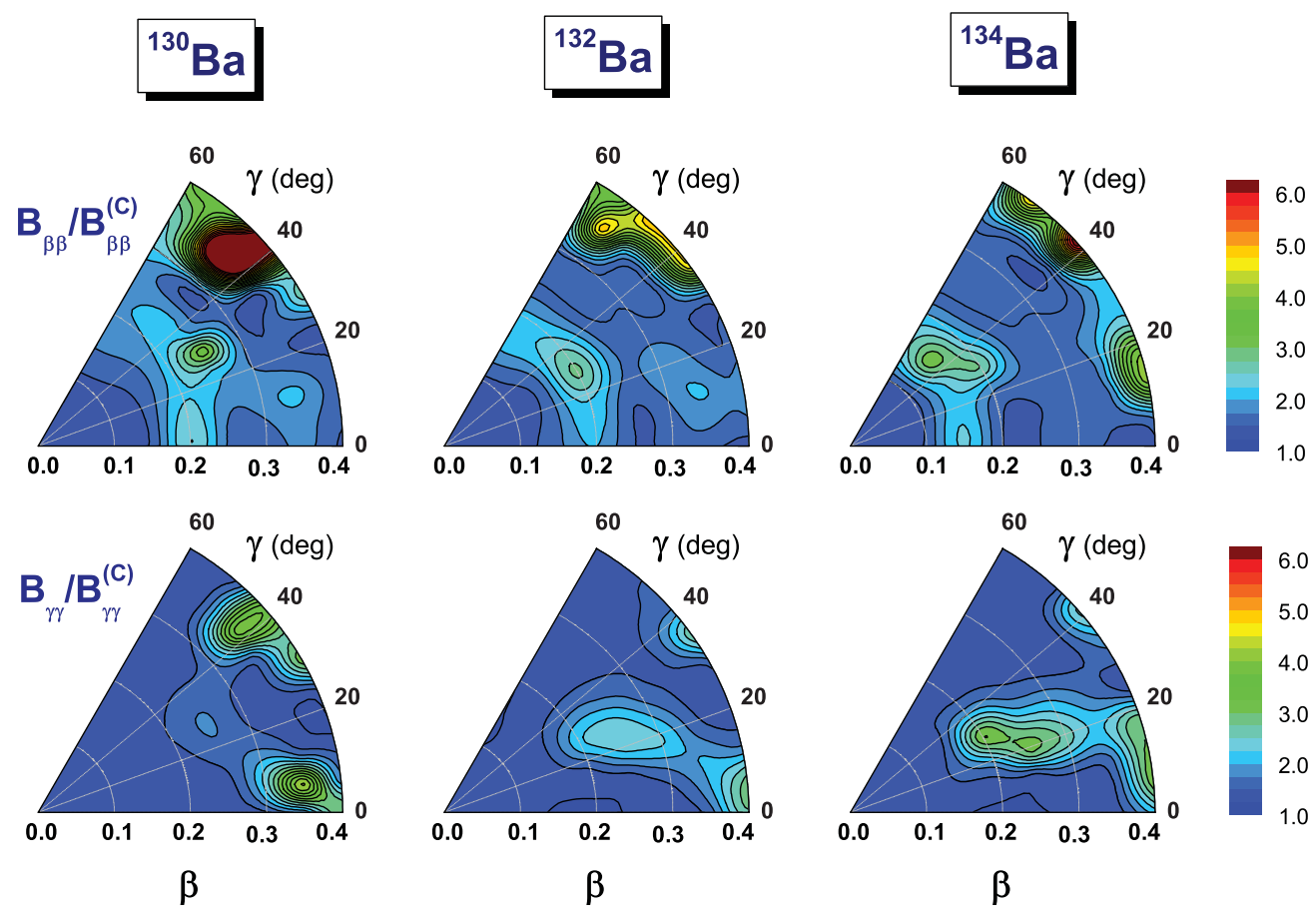

FIG. 6. (Color online) Same as described in the caption to Fig. 4 but for ${ }^{130-134}$ Ba.

neither the monopole pairing nor the quadrupole particle-hole interaction contribute to the time-odd mean fields, that is, only the quadrupole-pairing interaction induces the time-odd contribution in the $P+Q$ model. The reason for using the $P+Q$ Hamiltonian as an intermediate step is that CHFB + LQRPA calculations cannot yet be performed in the full configuration space of occupied and unoccupied self-consistent mean-field single-particle (quasiparticle) states. The RHB self-consistent solutions will therefore be used to determine the parameters of the corresponding $P+Q$ Hamiltonian, and the CHFB + LQRPA calculation of the deformation-dependent moments of inertia and mass parameters will be carried out in the restricted configuration space of two major oscillator shells for neutrons $\left(N_{\mathrm{sh}}=4,5\right)$ and protons $\left(N_{\mathrm{sh}}=3,4\right)[53]$.

Starting from the fully self-consistent RHB calculation, based on the relativistic functional $D D-P C 1$ [55] and the separable pairing interaction [56,57] (cf. Fig. 1), the parameters of the corresponding $P+Q$ Hamiltonian are determined as follows. The spherical single-particle energies correspond to the RHB single-particle energies at zero deformation (spherical configurations) scaled by the corresponding effective mass $\left(m^{*} / m=0.71\right.$ for the functional $\left.D D-P C 1\right)$. The neutron and proton monopole pairing strengths are adjusted to reproduce the average pairing gaps at spherical configuration obtained in the RHB calculation. The strength of the quadrupole particle-hole interaction is adjusted to reproduce the axial quadrupole deformation of the potential minimum of the RHB self-consistent solution. Finally, the neutron and proton quadrupole pairing strengths are evaluated at the spherical configuration using the self-consistent prescription of the authors of Ref. [67].

For the self-consistent RHB quadrupole constrained energy maps of $\mathrm{Xe}$ and $\mathrm{Ba}$ isotopes shown in Fig. 1, the corresponding energy surfaces calculated with the $P+Q$ Hamiltonian are plotted in Fig. 2. One notices that the $P+Q$ energy surfaces reproduce the isotopic evolution of $\beta$ and $\gamma$ softness predicted by the RHB self-consistent calculation, especially in the nearly flat regions around the minimum that determine the low-energy excitation spectra.

Using the solutions obtained with the $P+Q$ Hamiltonian, the CHFB + LQRPA method was used to calculate the collective inertia parameters, as described in the previous section. At the same time we also calculated the IB moments of inertia and cranking mass parameters based on the same CHFB states. The ratios of the moments of inertia, calculated with and without the contribution of time-odd mean fields and the corresponding ratios for the mass parameters, are plotted in Figs. 3 and 4, respectively, for the three Xe nuclei and in Figs. 5 and 6 for the $\mathrm{Ba}$ isotopes.

In the region of interest in the $\beta-\gamma$ plane, that is, close to the minima of the constrained energy surfaces, the ratios of the LQRPA and IB moments of inertia $\left(B_{x}, B_{y}, B_{z}\right)$ range between $\approx 1.1$ and $\approx 1.4$. The dependence on $\beta$ and $\gamma$ is different for the moments of inertia that correspond to three the intrinsic axes. For each moment of inertia the deformation dependence is very similar in all six $\mathrm{Xe}$ and $\mathrm{Ba}$ isotopes considered in the present study. The pattern for the ratios of the collective masses $B_{\beta \beta}$ and $B_{\gamma \gamma}$ appears to be more complex. In general the ratios range from 1 to 2 , however, at some deformation points one finds peaks for which the LQRPA values are more than five times larger than the cranking masses. In these regions avoided crossings in the LQRPA frequencies as functions of quadrupole deformations occur between one of the collective modes and a noncollective mode (usually these correspond to the second and third lowest LQRPA modes). The components of the two LQRPA modes mix in regions 

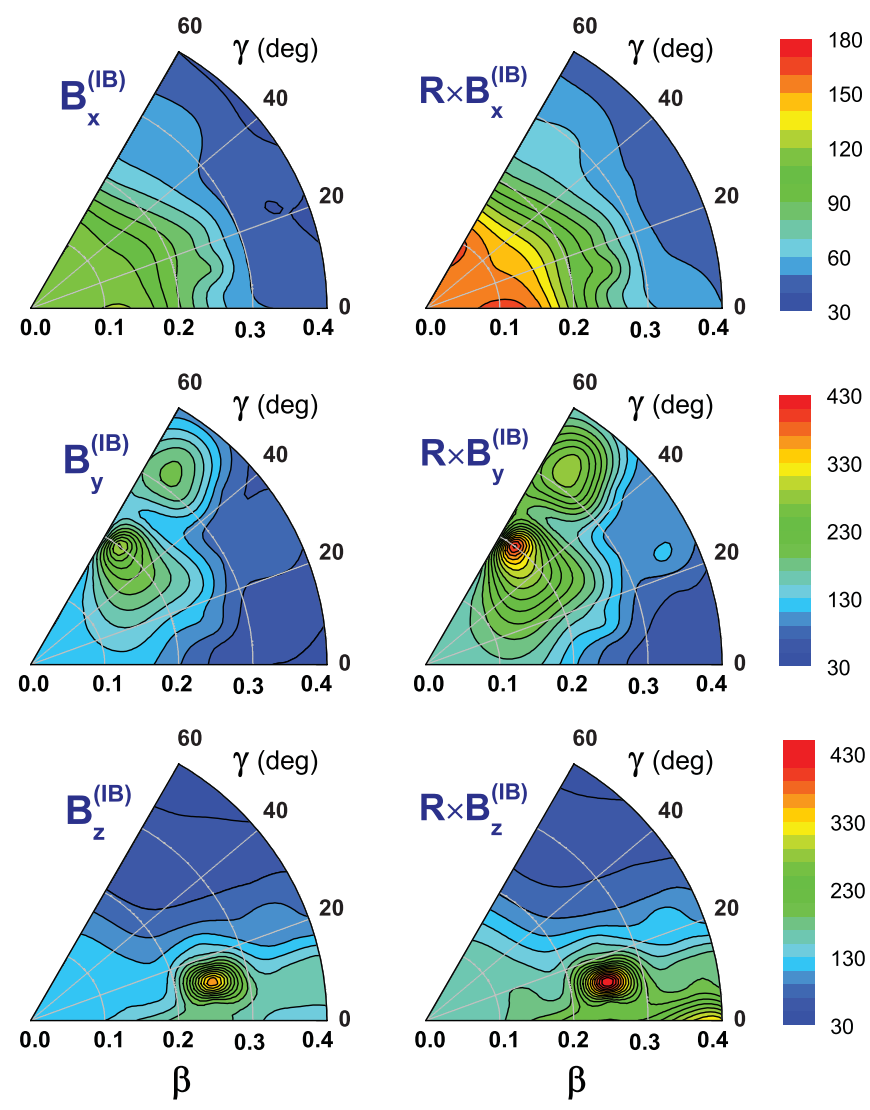

FIG. 7. (Color online) The IB moments of inertia for ${ }^{128} \mathrm{Xe}$ calculated from Eq. (29) using the fully self-consistent RHB quasiparticle states (left column) and multiplied by the CHFB + LQRPA ratios that represent the contribution of time-odd mean fields (right column).

of such crossings and quadrupole collectivity weakens. This results in the large collective LQRPA mass (large inertia in the quadrupole direction).

Taking as an example the nucleus ${ }^{128} \mathrm{Xe}$, in Figs. 7 and 8 we illustrate the effect of including time-odd mean fields on the moments of inertia and mass parameters based on constrained RHB mean-field solutions, respectively. In the left column of Fig. 7 we display the IB moments of inertia calculated from Eq. (29) using the fully self-consistent RHB quasiparticle states, and the corresponding cranking mass parameters $B_{\beta \beta}^{(C)}$ and $B_{\gamma \gamma}^{(C)}$ [Eq. (30)] are shown in the left column of Fig. 8. The contribution of the time-odd mean fields is then approximately taken into account by multiplying the IB moments of inertia and cranking masses by the corresponding factors determined using the CHFB + LQRPA method (Figs. 3 and 4 , respectively). The results are shown in the right columns of Fig. 7 (moments of inertia) and Fig. 8 (vibrational mass parameters). The overall effect of the time-odd mean fields on the moments of inertia appears to be a simple enhancement of the IB values, almost independent of the deformations $\beta$ and $\gamma$. Both the value and the deformation dependence of the cranking mass parameters $B_{\beta \beta}^{(C)}$ and $B_{\gamma \gamma}^{(C)}$ are strongly modified by the contribution of the time-odd mean fields.
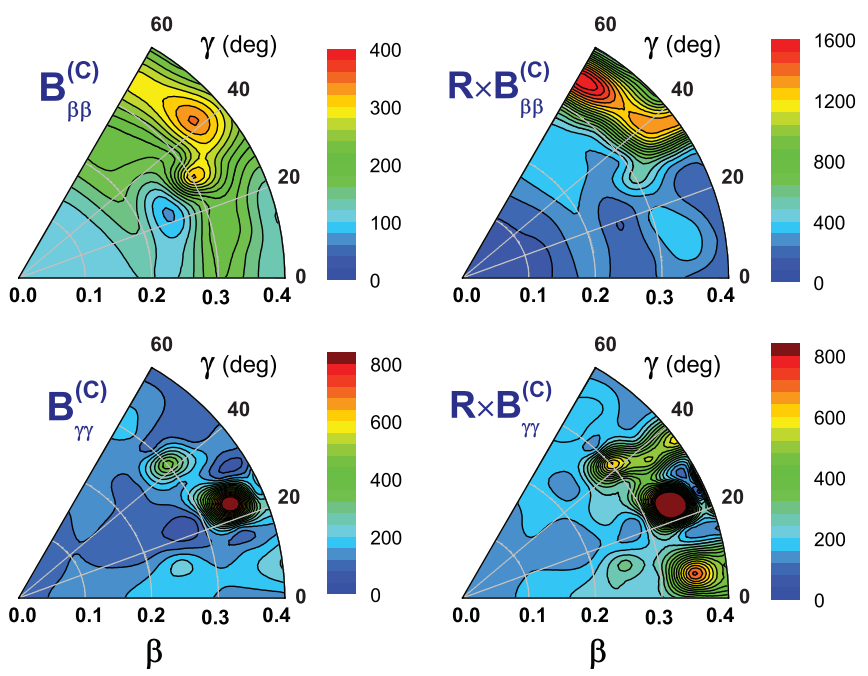

FIG. 8. (Color online) The cranking mass parameters $B_{\beta \beta}^{(C)}$ and $B_{\gamma \gamma}^{(C)}$ [Eq. (30)] for ${ }^{128} \mathrm{Xe}$, based on the self-consistent RHB ground-state solution (left column) and multiplied by the CHFB + LQRPA ratios that represent the contribution of time-odd mean fields (right column).

\section{B. Low-energy spectra and $\boldsymbol{E} 2$ transitions}

The corresponding low-energy spectra and transition probabilities obtained by diagonalization of the five-dimensional collective Hamiltonian are shown in Figs. 9 and 10 for ${ }^{128-132} \mathrm{Xe}$ and ${ }^{130-134} \mathrm{Ba}$, respectively, in comparison to the available data. In the left columns we display the excitation spectra obtained with the IB moments of inertia Eq. (29) and cranking mass parameters Eq. (30), calculated starting from constrained self-consistent RHB mean-field solutions. Obviously both the moments of inertia and the vibrational mass parameters are considerably reduced compared to the empirical values. This is reflected in the stretching of the theoretical ground-state bands and in the predicted high excitation energies of the states $0_{2}^{+}, 0_{3}^{+}$, and structures built on $2_{2}^{+}$. The agreement with the experiment is dramatically improved by the approximate treatment of time-odd mean fields using the CHFB + LQRPA method. The spectra shown in the middle panels are obtained by multiplying the IB moments of inertia and cranking mass parameters of the collective Hamiltonian by the corresponding deformationdependent factors determined using the CHFB + LQRPA (cf. Figs. 3 and 4). The increase of the moments of inertia leads to a compression of the (quasi)rotational bands and, in particular, this effect is clearly observed for the yrast sequences shown in Figs. 9 and 10. The larger values of the vibrational masses result in lowering the excited bandheads, that is, the states $2_{2}^{+}$, as well as the excited $0^{+}$states. The transition probabilities are slightly reduced by the contribution of time-odd mean fields. However, both the excitation energies and transition rates are in very good agreement with the experiment.

There are, of course, additional effects not taken into account in our hybrid approach. First of all we have not included the time-odd contribution to the mass parameter $B_{\beta \gamma}$. The diagonal mass parameters $B_{\beta \beta}$ and $B_{\gamma \gamma}$ are positivedefinite and the ratios of their values with respect to the 

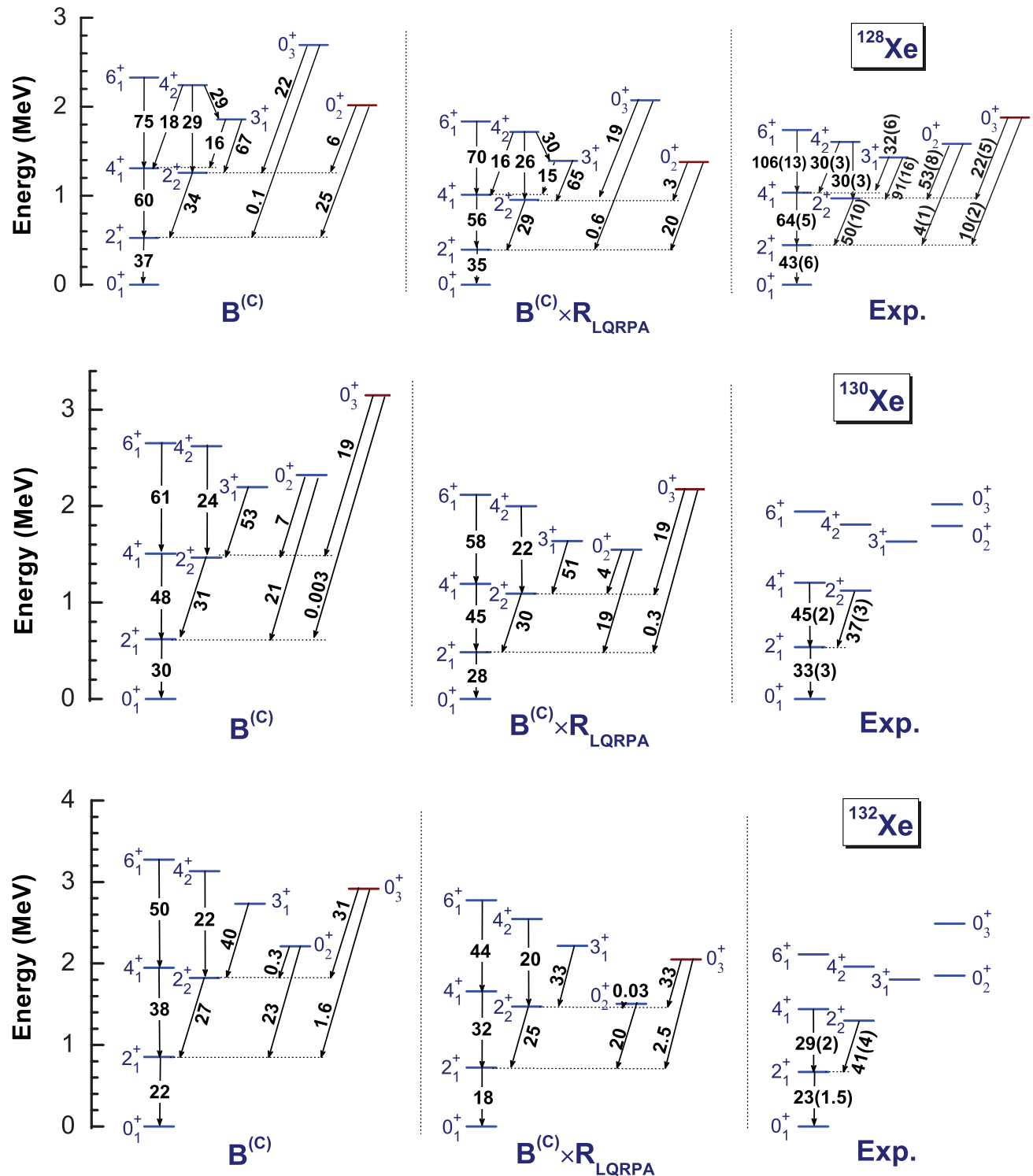

FIG. 9. (Color online) Low-energy level schemes of ${ }^{128-132} \mathrm{Xe}$ calculated with the five-dimensional collective Hamiltonian, in comparison to available data (right column). The spectra are calculated with the IB moments of inertia and cranking mass parameters (left column) and with the approximate inclusion of the contribution of time-odd mean fields using the CHFB + LQRPA method (middle column). $B(E 2)$ values are given in Weisskopf units.

cranking mass parameters are well defined for all points of the $\beta-\gamma$ plane. However, the off-diagonal mass parameter $B_{\beta \gamma}$ changes sign in the $\beta-\gamma$ plane and vanishes for points at the $\gamma=0$ and $\gamma=\pi / 3$ axes. Since in general the points in the $\beta-\gamma$ plane where the values of the LQRPA $B_{\beta \gamma}$ vanish are different from those for which the corresponding cranking mass parameter is zero, the ratio of these two quantities does not provide any useful information for the discussion of the effect of time-odd mean fields. Therefore we note that the spectra shown in the middle columns of Figs. 9 and 10 were also calculated with the cranking mass parameter $B_{\beta \gamma}$ and, in principle, this will affect the couplings between the $\beta$ and $\gamma$ vibrational modes.

Concerning the excitation energies of the $0^{+}$states, we note that an effect that is not included in the present model is the coupling of nuclear shape oscillations with pairing vibrations (i.e., vibrations of the pairing density). A number of studies have shown that excited $0^{+}$states are very sensitive to the coupling between these modes. For the $\mathrm{Ba}$ and $\mathrm{Xe}$ isotopes, in particular, the effect of coupling between pairing vibrations and axial quadrupole vibrations was analyzed by the authors of Ref. [68]. It was pointed out that this coupling in general leads to a pronounced lowering of excited $0^{+}$states, bringing them in much closer agreement to the data.

\section{SUMMARY}

One of the most important current research topics in nuclear structure theory is the development of a fully microscopic 

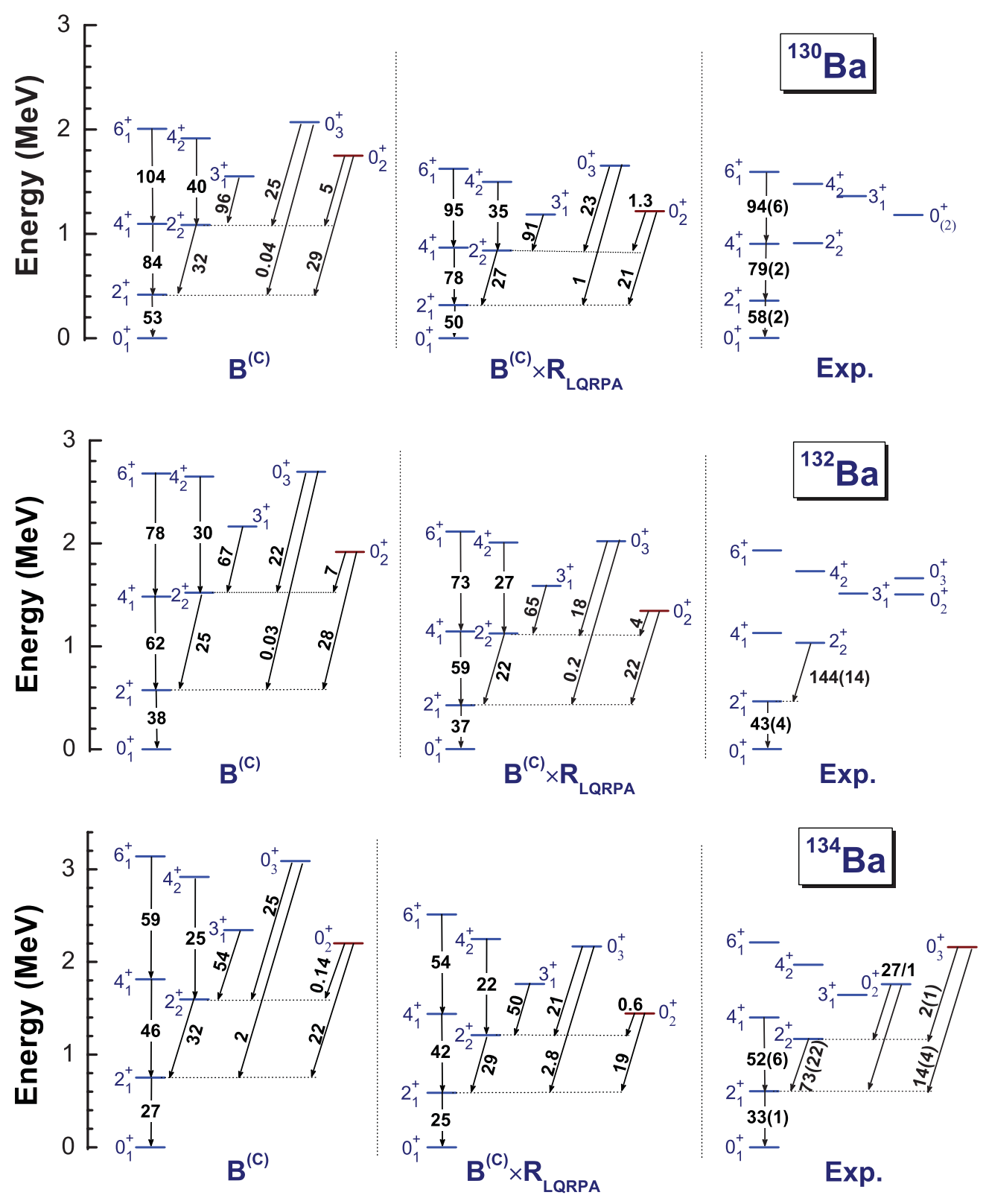

FIG. 10. (Color online) Same as described in the caption to Fig. 9 but for ${ }^{130-134} \mathrm{Ba}$.

framework, based on universal EDFs, which will provide a quantitative description of phenomena related to the evolution of shell structure from stable nuclei to exotic systems far from stability. To describe complex collective excitation spectra and transition rates such a framework must be extended beyond its basic level of implementation-the self-consistent mean-field-and include collective correlations related to restoration of broken symmetries and fluctuations in collective coordinates.

Several microscopic models have recently been developed, based on the Gogny effective interaction, Skyrme functionals, and relativistic density functionals, that employ the angularmomentum (and particle-number) projected GCM to calculate collective excited states and transition probabilities. The numerical implementation of these models, however, is rather complex and applications to medium-heavy and heavy nuclei, especially in the case of $\gamma$-soft or triaxial shapes, are computationally very demanding and time consuming. Additional issues arise from dealing with transition densities rather than one-body densities that correspond to a single product state. An alternative approach that avoids most of the problems associated with symmetry restoration when using transition densities, and takes into account collective correlations in a self-consistent way, is based on the quadrupole collective Hamiltonian with deformation-dependent vibrational inertial functions and moments of inertia. Because of computational complexity, however, in most current implementations of the collective Hamiltonian model the moments of inertia and mass parameters do not include the contributions of timeodd mean fields (the so-called Thouless-Valatin dynamical 
rearrangement contributions), and this breaks the selfconsistency of the approach.

Using an efficient microscopic method of deriving the fivedimensional collective Hamiltonian, based on the adiabatic self-consistent collective coordinate approach, in this work we developed a hybrid model to analyze the contribution of the Thouless-Valatin contributions to the moments of inertia and mass parameters of a collective Hamiltonian based on microscopic relativistic EDFs. Starting from the fully self-consistent constrained RHB calculations of triaxial energy surfaces in the $\beta-\gamma$ plane, the inertial functions of the collective Hamiltonian are calculated in the cranking approximation. At the same time the self-consistent mean-field solutions are used to determine the parameters of an equivalent pairing-plus-quadrupole $(P+Q)$ Hamiltonian, that is used as a basis for the CHFB plus LQRPA calculation of the deformation-dependent corrections to the IB moments of inertia and cranking mass parameters.

In the present analysis we use the hybrid model based on the microscopic collective Hamiltonian and the CHFB + LQRPA method to estimate the contribution of time-odd mean fields (Thouless-Valatin contribution) to the moments of inertia and mass parameters and investigate their effect on the collective excitation spectra of $\gamma$-soft Xe and Ba nuclei. It has been shown that the low-energy excitation spectra obtained with the IB moments of inertia and cranking mass parameters, calculated starting from constrained self-consistent RHB mean-field solutions, are only in qualitative agreement with the data: the calculated ground-state bands are stretched compared to experiment and the energies of excited $0^{+}$states, as well as structures built on $2_{2}^{+}$, are considerably higher than their experimental counterparts. This means that the IB moments of inertia and the cranking mass parameters are markedly reduced compared to the empirical values.

In the second step the CHFB + LQRPA method was used to calculate the ratios of the moments of inertia, calculated with and without the contribution of time-odd mean fields, and the corresponding ratios for the mass parameters. In the region of interest in the $\beta-\gamma$ plane, that is, close to the minima of the constrained energy surfaces, the ratios of the LQRPA and IB moments of inertia range between $\approx 1.1$ and $\approx 1.4$, with a weak dependence on the deformation parameters $\beta$ and $\gamma$. The pattern for the ratios of the collective masses is more complex and, in general, the values range from 1 to 2 . The resulting low-energy spectra and transition probabilities determined by diagonalization of the five-dimensional collective Hamiltonian for ${ }^{128-132} \mathrm{Xe}$ and ${ }^{130-134} \mathrm{Ba}$ were compared to the available data. The excitation spectra obtained by multiplying the IB moments of inertia and cranking mass parameters of the collective Hamiltonian by the corresponding deformationdependent factors, determined using the CHFB + LQRPA method, are in much better agreement with experiment. The enhancement of the moments of inertia leads to a compression of the (quasi)rotational bands and the larger values of the vibrational masses result in lowering the excited bandheads. The transition probabilities are slightly reduced by the contribution of time-odd mean fields, but still in very good agreement with empirical values.

For a quadrupole collective Hamiltonian based on microscopic self-consistent calculations of constrained binding energy surfaces, the present study shows, on a quantitative level, the importance of including contributions of time-odd mean fields to the moments of inertia and collective mass parameters and their effect on low-energy excitation spectra and transition rates. In the hybrid model the potential energy surface is determined by constrained self-consistent RHB mean-field solutions, and this presents a major improvement compared to previous studies that used the CHFB + LQRPA approach based on the $P+Q$ model $[53,59,60]$. Although we show that the inertial parameters are significantly modified by time-odd mean fields, the present hybrid model does not fully take into account their effects. Work is currently underway to include, in a fully consistent way, the Thouless-Valatin dynamical rearrangement contributions to the moments of inertia and mass parameters of the collective Hamiltonian built on the RHB model.

\section{ACKNOWLEDGMENTS}

This work was supported in part by the JSPS Grant-in-Aid for Scientific Research (Grants No. 20105003 and No. 21340073), the NSFC under Grant No. 11105110, the Southwest University Initial Research Foundation Grant to Doctor (Grant No. SWU110039), the Fundamental Research Funds for the Central Universities (XDJK2011B002), and MZOS - Project No. 1191005-1010. The work of T. Nikšić and D. Vretenar was supported in part by the Chinese-Croatian project "Nuclear structure and astrophysical applications." T. Nikšić and Z. P. Li acknowledge support by the Croatian National Foundation for Science. N. Hinohara was supported by the RIKEN Special Postdoctoral Researcher Program. Calculations were partially carried out at the RIKEN Cluster of Clusters (RICC) facility. Discussions during the YIPQS international workshop on "Dynamics and Correlations in Exotic Nuclei 2011" were useful to complete this work.
[1] Extended Density Functionals in Nuclear Structure Physics, Lecture Notes in Physics 641, edited by G. A. Lalazissis, P. Ring, and D. Vretenar (Springer, Heidelberg, Germany, 2004).

[2] M. Bender, P.-H. Heenen, and P.-G. Reinhard, Rev. Mod. Phys. 75, 121 (2003).

[3] D. Vretenar, A. V. Afanasjev, G. A. Lalazissis, and P. Ring, Phys. Rep. 409, 101 (2005).

[4] J. Dobaczewski, J. Phys.: Conf. Ser. 312, 092002 (2011).
[5] R. Rodríguez-Guzmán, J. L. Egido, and L. M. Robledo, Phys. Rev. C 65, 024304 (2002).

[6] R. Rodríguez-Guzmán, J. L. Egido, and L. M. Robledo, Nucl. Phys. A 709, 201 (2002).

[7] R. Rodríguez-Guzmán, J. L. Egido, and L. M. Robledo, Phys. Rev. C 69, 054319 (2004).

[8] T. R. Rodríguez and J. L. Egido, Phys. Rev. Lett. 99, 062501 (2007).

[9] T. R. Rodríguez and J. L. Egido, Phys. Lett. B 663, 49 (2008). 
[10] T. R. Rodríguez and J. L. Egido, Phys. Rev. C 81, 064323 (2010).

[11] A. Valor, P.-H. Heenen, and P. Bonche, Nucl. Phys. A 671, 145 (2000).

[12] M. Bender, H. Flocard, and P.-H. Heenen, Phys. Rev. C 68, 044321 (2003).

[13] T. Duguet, M. Bender, P. Bonche, and P.-H. Heenen, Phys. Lett. B 559, 201 (2003).

[14] M. Bender, P. Bonche, T. Duguet, and P.-H. Heenen, Phys. Rev. C 69, 064303 (2004).

[15] M. Bender, P. Bonche, and P.-H. Heenen, Phys. Rev. C 74, 024312 (2006).

[16] M. Bender and P.-H. Heenen, Phys. Rev. C 78, 024309 (2008).

[17] T. Nikšić, D. Vretenar, and P. Ring, Phys. Rev. C 73, 034308 (2006).

[18] T. Nikšić, D. Vretenar, and P. Ring, Phys. Rev. C 74, 064309 (2006).

[19] J. M. Yao, J. Meng, P. Ring, and D. Pena Arteaga, Phys. Rev. C 79, 044312 (2009).

[20] J. M. Yao, J. Meng, P. Ring, and D. Vretenar, Phys. Rev. C 81, 044311 (2010).

[21] M. Anguiano, J. L. Egido, and L. M. Robledo, Nucl. Phys. A 696, 467 (2001).

[22] J. Dobaczewski, M. V. Stoitsov, W. Nazarewicz, and P.-G. Reinhard, Phys. Rev. C 76, 054315 (2007).

[23] T. Duguet and J. Sadoudi, J. Phys. G: Nucl. Part. Phys. 37, 064009 (2010).

[24] D. Lacroix, T. Duguet, and M. Bender, Phys. Rev. C 79, 044318 (2009).

[25] M. Bender, T. Duguet, and D. Lacroix, Phys. Rev. C 79, 044319 (2009).

[26] P.-G. Reinhard and K. Goeke, Rep. Prog. Phys. 50, 1 (1987).

[27] P. Bonche, J. Dobaczewski, H. Flocard, P.-H. Heenen, and J. Meyer, Nucl. Phys. A 510, 466 (1990).

[28] L. Próchniak and S. G. Rohoziński, J. Phys. G 36, 123101 (2009).

[29] P. Ring and P. Schuck, The Nuclear Many-Body Problem (Springer-Verlag, Heidelberg, Germany, 1980).

[30] B. Giraud and B. Grammaticos, Nucl. Phys. A 255, 141 (1975).

[31] M. Girod and B. Grammaticos, Nucl. Phys. A 330, 40 (1979).

[32] M. Baranger and M. Vénéroni, Ann. Phys. (NY) 114, 123 (1978).

[33] F. Villars, Nucl. Phys. A 285, 269 (1977).

[34] G. Do Dang, A. Klein, and N. R. Walet, Phys. Rep. 335, 93 (2000).

[35] T. Nakatsukasa, N. R. Walet, and G. D. Dang, Phys. Rev. C 61, 014302 (1999).

[36] N. Hinohara, T. Nakatsukasa, M. Matsuo, and K. Matsuyanagi, Prog. Theor. Phys. 117, 451 (2007).
[37] M. Matsuo, T. Nakatsukasa, and K. Matsuyanagi, Prog. Theor. Phys. 103, 959 (2000).

[38] D. J. Thouless and J. G. Valatin, Nucl. Phys. 31, 211 (1962).

[39] K. Goeke and P.-G. Reinhard, Ann. Phys. (NY) 124, 249 (1980).

[40] R. E. Peierls and J. Yoccoz, Proc. Phys. Soc. A 70, 381 (1957).

[41] D. R. Inglis, Phys. Rev. 103, 1786 (1956).

[42] J. Libert, M. Girod, and J.-P. Delaroche, Phys. Rev. C 60, 054301 (1999).

[43] E. Clément et al., Phys. Rev. C 75, 054313 (2007).

[44] L. Próchniak, P. Quentin, D. Samsoen, and J. Libert, Nucl. Phys. A 730, 59 (2004).

[45] J.-P. Delaroche, M. Girod, J. Libert, H. Goutte, S. Hilaire, S. Péru, N. Pillet, and G. F. Bertsch, Phys. Rev. C 81, 014303 (2010).

[46] L. Próchniak and P. Ring, Int. J. Mod. Phys. E 13, 217 (2004).

[47] T. Nikšić, Z. P. Li, D. Vretenar, L. Próchniak, J. Meng, and P. Ring, Phys. Rev. C 79, 034303 (2009).

[48] Z. P. Li, T. Nikšić, D. Vretenar, J. Meng, G. A. Lalazissis, and P. Ring, Phys. Rev. C 79, 054301 (2009).

[49] Z. P. Li, T. Nikšić, D. Vretenar, and J. Meng, Phys. Rev. C 80, 061301 (2009).

[50] Z. P. Li, T. Nikšić, D. Vretenar, and J. Meng, Phys. Rev. C 81, 034316 (2010).

[51] Z. P. Li, T. Nikšić, D. Vretenar, P. Ring, and J. Meng, Phys. Rev. C 81, 064321 (2010).

[52] N. Hinohara, T. Nakatsukasa, M. Matsuo, and K. Matsuyanagi, Prog. Theor. Phys. 115, 567 (2006).

[53] N. Hinohara, K. Sato, T. Nakatsukasa, M. Matsuo, and K. Matsuyanagi, Phys. Rev. C 82, 064313 (2010).

[54] W. Pauli, in Handbuch der Physik, Vol. XXIV (Springer-Verlag, Berlin, 1933), p. 120.

[55] T. Nikšić, D. Vretenar, and P. Ring, Phys. Rev. C 78, 034318 (2008).

[56] Y. Tian, Z. Ma, and P. Ring, Phys. Lett. B 676, 44 (2009).

[57] T. Nikšić, P. Ring, D. Vretenar, Y. Tian, and Z. Y. Ma, Phys. Rev. C 81, 054318 (2010).

[58] J. Dobaczewski and J. Skalski, Nucl. Phys. A 369, 123 (1981).

[59] K. Sato and N. Hinohara, Nucl. Phys. A 849, 53 (2011).

[60] N. Hinohara and Y. Kanada-En'yo, Phys. Rev. C 83, 014321 (2011)

[61] K. Yoshida and N. Hinohara, Phys. Rev. C 83, 061302 (2011).

[62] M. Baranger and K. Kumar, Nucl. Phys. 62, 113 (1965).

[63] M. Baranger and K. Kumar, Nucl. Phys. A 110, 490 (1968).

[64] K. Kumar and M. Baranger, Nucl. Phys. 110, 529 (1968).

[65] M. Baranger and K. Kumar, Nucl. Phys. A 122, 241 (1968).

[66] K. Kumar and M. Baranger, Nucl. Phys. A 122, 273 (1968).

[67] H. Sakamoto and T. Kishimoto, Phys. Lett. B 245, 321 (1990).

[68] S. Pilat and K. Pomorski, Nucl. Phys. A 554, 413 (1993). 